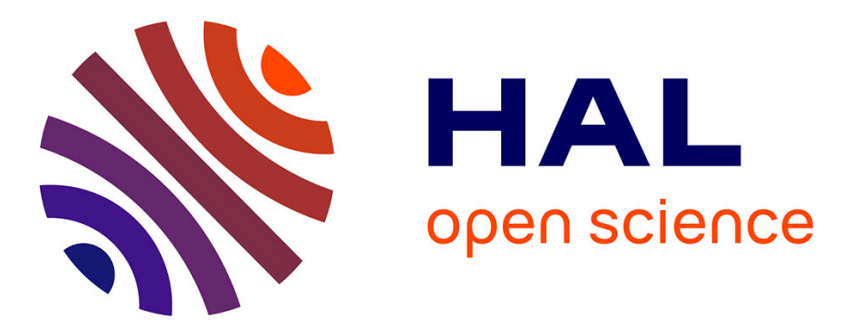

\title{
Potentialities of an Electro-Optic Crystal Fed by Nuclear Magnetic Resonant Coil for Remote and Low-Invasive Magnetic Field Characterization
}

Reina Aydé, Gwenaël Gaborit, Pierre Jarrige, Lionel Duvillaret, R. Sablong, Anne-Laure Perrier, O. Beuf

\section{To cite this version:}

Reina Aydé, Gwenaël Gaborit, Pierre Jarrige, Lionel Duvillaret, R. Sablong, et al.. Potentialities of an Electro-Optic Crystal Fed by Nuclear Magnetic Resonant Coil for Remote and LowInvasive Magnetic Field Characterization. IEEE Sensors Journal, 2013, 13 (4), pp.1274 - 1280. 10.1109/JSEN.2012.2230623 . hal-01814413

\section{HAL Id: hal-01814413 \\ https://hal.univ-smb.fr/hal-01814413}

Submitted on 18 Jun 2018

HAL is a multi-disciplinary open access archive for the deposit and dissemination of scientific research documents, whether they are published or not. The documents may come from teaching and research institutions in France or abroad, or from public or private research centers.
L'archive ouverte pluridisciplinaire HAL, est destinée au dépôt et à la diffusion de documents scientifiques de niveau recherche, publiés ou non, émanant des établissements d'enseignement et de recherche français ou étrangers, des laboratoires publics ou privés. 


\title{
Potentialities of an Electro-Optic Crystal Fed by Nuclear Magnetic Resonant Coil for Remote and Low-Invasive Magnetic Field Characterization
}

\author{
Reina Aydé, Gwenaël Gaborit, Pierre Jarrige, Lionel Duvillaret, \\ Raphaël Sablong, Anne-Laure Perrier, and Olivier Beuf
}

\begin{abstract}
In this paper, we demonstrate the use of a $\mathrm{LiTaO}_{3}$ crystal associated with a typical nuclear magnetic resonant loop coil to perform an optically remote radio frequency magnetic-field characterization. The whole transduction scheme is theoretically and experimentally studied. The measurement dynamics reaches $60 \mathrm{~dB}$. The minimum detectable magnetic field is lower than $1 \mathrm{nT}$, which corresponds to an induced inner crystal electric field as low as $30 \mathrm{mV} / \mathrm{m}$. To evaluate the spatial potentialities of the sensor, a 1-D mapping of the field along an asymmetric butterfly-shaped loop coil is performed. The result is in good agreement with finite-difference time-domain simulations and demonstrates the vectorial behavior of the sensor device.
\end{abstract}

Index Terms-Electro-optics, magnetic field sensor, magnetic resonance imaging, nuclear magnetic resonance.

\section{INTRODUCTION}

$\mathbf{M}$ AGNETIC resonance imaging (MRI) is based on a spatial characterization of the nuclear magnetization. Recording the variation of the nuclear magnetization is often realized with a MRI external surface coil. However, spatial resolution and image quality achievable with external surface coils are limited in the case of thin internal organs, e.g. bowel. On the other hand, the use of an endoluminal MRI coil located close to the area of interest could provide both high contrast to noise ratio (CNR) and high spatial resolution images leading, in turn, to a good evaluation of biological diseases [1], [2].

Manuscript received October 12, 2012; revised November 20, 2012; accepted November 21, 2012. Date of publication November 29, 2012; date of current version February 11, 2013. This work was supported in part by the Region Rhone Alpes, the DGA (French Military Programs Management and Procurement Agency - Project RAPID SNIFER) and DGCIS. The associate editor coordinating the review of this paper and approving it for publication was Dr. Chang-Soo Kim.

R. Aydé, R. Sablong, A.-L. Perrier, and O. Beuf are with Université de Lyon, CREATIS, CNRS UMR 5220, Université Lyon 1, Villeurbanne 69239, France (e-mail: reina.ayde@creatis.insa-lyon.fr; raphael.sablong@ univ-lyon1.fr; anne-laure.perrier@univ-lyon1.fr; olivier.beuf@univ-lyon1.fr).

G. Gaborit and P. Jarrige are with IMEP-LAHC, UMR CNRS 5130, Université de Savoie, Le Bourget du Lac 73 376, France, and also with Kapteos, Bat.Chablais, Rue Lac de Thuile, Le Bourget du Lac 73 376, France (e-mail: gwenael.gaborit@univ-savoie.fr; pierre.jarrige@ kapteos.com).

L. Duvillaret is with Kapteos, Bat.Chablais, Rue Lac de Thuile, Le Bourget du Lac 73 376, France (e-mail: lionel.duvillaret@kapteos.com).

Color versions of one or more of the figures in this paper are available online at http://ieeexplore.ieee.org.

Digital Object Identifier 10.1109/JSEN.2012.2230623
Since metallic coaxial cables are mainly used with endoluminal coils, also located within the MRI transmit RF coil, heating phenomena may occur inside biological media [3]. Optical fibre link can be used to overcome heating problem, thus ensuring patients safety [4], [5]. Direct modulation of a laser diode remains invasive because its biasing requires a DC power supply [4]. Furthermore, the achieved realizations including active devices may also be disturbed by the static magnetic field [4], [6], [7].

Magnetic field characterization involving passive optical system has already been performed [8]-[11]. This type of magnetic probe is based on an Electro-Optic (EO) material associated to a magnetic loop inducing an electric (E)-field proportional to a magnetic field component. This experimental configuration allows to minimize the invasiveness: the probe can be pigtailed, does not need power supply and includes metallic element much shorter than the wavelength of the field to be measured. This kind of sensor has been studied by Suzuki et al. [8] and exploits a magnetic loop double loaded with $\mathrm{LiNbO}_{3}$ crystal, acting as the capacitance of the resonant equivalent circuit. The realized set-up is dedicated to measure the magnetic field associated to a microwave electromagnetic field propagated along a stripline. We here propose a similar configuration, dedicated to MRI based RF magnetic field measurement. Nevertheless, as our field of interest concerns proton MRI, the resonant frequency is precisely defined (e.g. $127 \mathrm{MHz}$ at $3 \mathrm{~T}$ ). Moreover, this resonant frequency is lower in MRI system compared to the microwave frequency probed by Suzuki et al., thus inducing a lower electromotive force $\varepsilon=\frac{d \phi}{d t}$ applied to the crystal. $\phi$ represents the magnetic flux. The loop coil is designed to be intrinsically resonant at $127 \mathrm{MHz}$ and the crystal capacitance is kept very low to keep the same frequency response. In the first section of the paper, the crystal orientation and the measurement set-up are studied and optimized in order to maximize the sensitivity, the linearity and the measurement dynamics. Then, the experimental setup used for the characterization of the EO magnetic probe is depicted. The response intensity of the probe is measured versus applied magnetic field amplitude. Finally, last section is dedicated to 1D magnetic field mapping of a butterfly-shaped loop RF coil. 


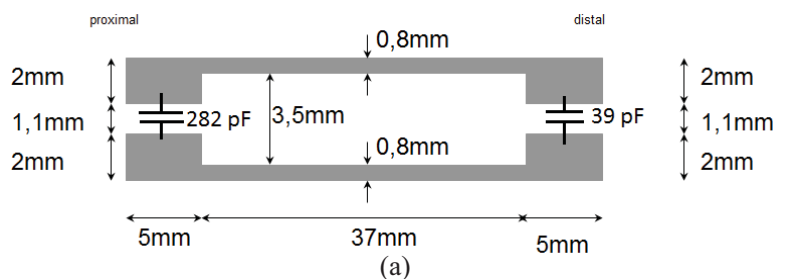

(a)

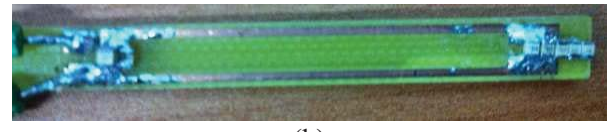

(b)

Fig. 1. Endoluminal RF coil. (a) Schematic. (b) Picture.

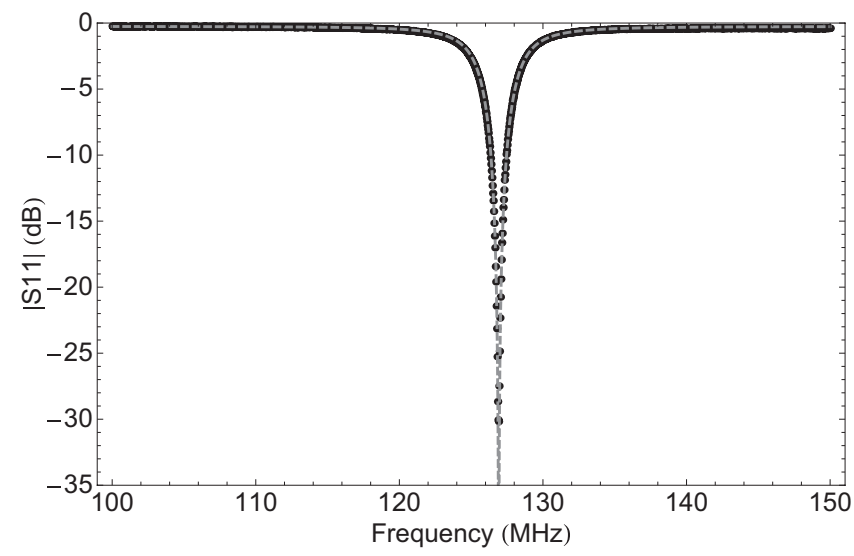

Fig. 2. Magnitude of the coil reflection coefficient. Black dots: measured with a vector network analyzer. Gray dashed line: measured with equivalent RLC circuit fitting curve.

\section{THEORETICAL BACKGROUND AND MEASUREMENT SET-UP}

\section{A. Endoluminal Coil Design}

Based on the previous study by Armenean et al. [1], we choose the parameter of the coil as those providing the best signal-to-noise ratio (SNR) for MRI together with suitable dimensions for endoluminal investigations. Hence, the coil has a rectangular shape with an external width set to $5.1 \mathrm{~mm}$, respecting medical constraints, and a length set to $47 \mathrm{~mm}$ providing good image penetration depth and exploration length. A $35 \mu$ m-thick copper coil is engraved on a insulating substrate FR4 $\left(\varepsilon_{r}=4\right.$ and thickness $\left.T=0.8 \mathrm{~mm}\right)$. A conductor width of $0.8 \mathrm{~mm}$ is defined to minimize ohmic losses then providing the best SNR images. The coil is tuned to a frequency of $127 \mathrm{MHz}$ and is matched at $50 \Omega$, using capacitors of $282 \mathrm{pF}$ and $39 \mathrm{pF}$ on distal and proximal part of the coil, respectively. A schematic and a picture of the endoluminal coil are presented in figure 1.

The characterization of the coil was performed thanks to a vector network analyzer and the magnitude of $\mathrm{S} 11$ reflection coefficient is given in figure 2. The resonant frequency is 126.9 $\mathrm{MHz}$ and its bandwidth is $3.5 \mathrm{MHz}$ at $-3 \mathrm{~dB}$.

The extracted parameters of the $R L C$ series equivalent circuit are $R=0.85 \Omega, L=46 \times 10^{-9} \mathrm{H}$ and $C=$ $34.2 \times 10^{-12} \mathrm{~F}$. These values are very close to the expected ones, obtained thanks to method of moments simulations.

\section{B. Electro-Optic Transducer}

The Pockels effect appears as an E-field induced modification of the eigen refractive indices in non-centrosymetric crystals. The developed transducer is based on an $x$-cut $\mathrm{LiTaO}_{3}$ crystal. An exhaustive analysis of the properties of EO crystals is described by Duvillaret et al. [12]. The EO tensor, which gives the vectorial dependence of the index ellipsoid with the E-field is the following one:

$$
\left(\begin{array}{lll}
0 & -r_{22} & r_{13} \\
0 & r_{22} & r_{13} \\
0 & 0 & r_{33} \\
0 & r_{51} & 0 \\
r_{51} & 0 & 0 \\
-r_{22} & 0 & 0
\end{array}\right) .
$$

This latter one leads to the refractive index ellipsoid equation (eq. 1):

$$
\begin{aligned}
& x^{2}\left(\frac{1}{n_{x}^{2}}-E_{y} r_{22}+E_{z} r_{13}\right)+y^{2}\left(\frac{1}{n_{y}^{2}}+E_{y} r_{22}+E_{z} r_{13}\right) \\
& +z^{2}\left(\frac{1}{n_{z}^{2}}+E_{z} r_{33}\right)+2 E_{y} r_{51} y z+2 E_{x} r_{51} x z-2 E_{x} r_{22} x y .
\end{aligned}
$$

A figure of merit can be calculated for this crystal [12]. As the crystal acts as a polarization state modulator, the relevant property is the E-field induced birefringence $\delta n=$ $n_{+}(\vec{E})-n_{-}(\vec{E})$. The EO figure of merit is depicted by the sensitivity vector $\overrightarrow{\Delta K}$, linked to the relative variation of refractive indices induced by the applied E field. This sensitivity vector is defined by:

$$
\overrightarrow{\Delta K}=\left.\vec{\nabla} \delta n(\vec{E})\right|_{\vec{E}=\overrightarrow{0}} .
$$

Its modulus gives the sensitivity of the EO crystal to the applied E field while its direction leads to the unique E-field component that will be probed. These properties are summarized by the following expression:

$$
\delta n(\vec{E}) \cong \delta n(\vec{E}=\overrightarrow{0})+\overrightarrow{\Delta K} \cdot \vec{E}
$$

A three-dimensional representation of $\overrightarrow{\Delta K}$ is given in figure 3 .

We here consider an $x$-cut $\mathrm{LiTaO}_{3}$, leading to a high sensitivity together with a transverse E-field probing (see Figure 3: $\Lambda=90^{\circ}$ ). For an applied $E$ field along $z$ axis, we get:

$$
\left|\overrightarrow{\Delta K}_{x-c u t, E_{z}}\right|=\frac{1}{2}\left(n_{z}^{3} r_{33}-n_{y}^{3} r_{13}\right)
$$

The theoretical value of $\left|\overrightarrow{\Delta K}_{x-c u t, E_{z}}\right|$ at the wavelength of $1550 \mathrm{~nm}$ is $120 \mathrm{pm} / \mathrm{V}$ assuming $n_{z}=2.116, n_{y}=2.117$, $r_{13}=7.5 \mathrm{pm} / \mathrm{V}$, and $r_{33}=33 \mathrm{pm} / \mathrm{V}$ [12].

The E-field induced dephasing between the two allowed polarization state inside the EO crystal writes:

$$
\Delta \varphi=\frac{2 \pi}{\lambda_{o p t}}\left|\overrightarrow{\Delta K}_{x-c u t, E_{z}}\right| \cdot E_{z} L_{x} .
$$

$\lambda_{\text {opt }}$ being the wavelength of the probing optical beam and $L_{x}$, the length of the crystal. 

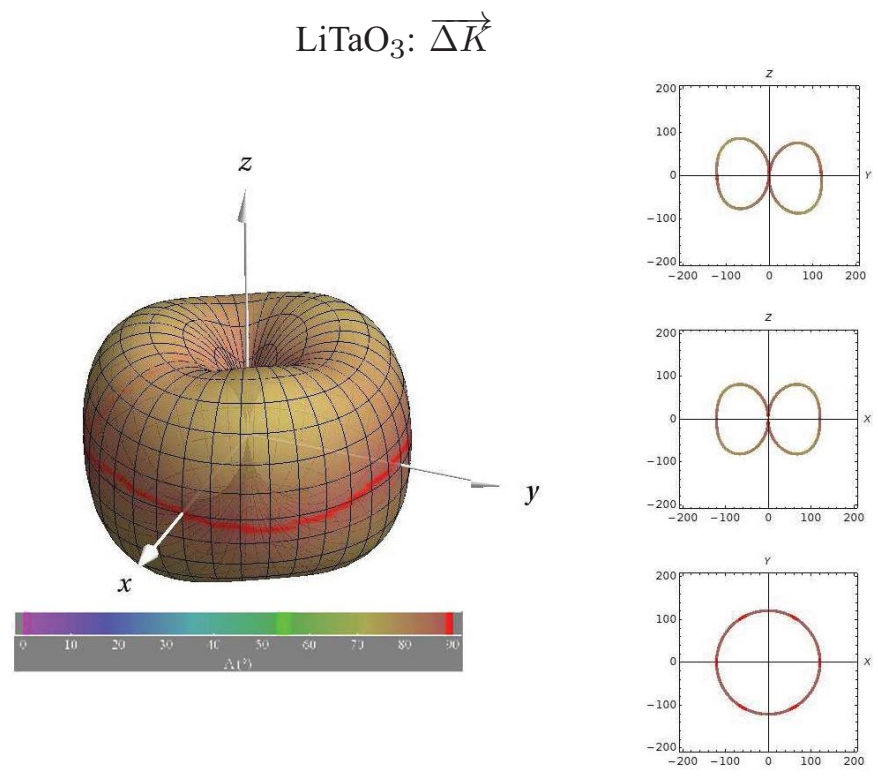

Fig. 3. $\mathrm{LiTaO}_{3}$ figure of merit for $\overrightarrow{\Delta K} \cdot|\overrightarrow{\Delta K}|$ is determined by the distance to the origin. Color: angle $\Lambda$ corresponding to the sensitivity axis orientation between $\overrightarrow{\Delta K}$ and the optical wave-vector $\vec{k}$. Scale at the bottom left. Projections onto the reference planes at the right side. The unit of $|\vec{K}|$ is $\mathrm{pm} \cdot \mathrm{V}^{-1}$.

\section{Measurement Set-Up}

The magnetic field source is constituted of a single wide band loop with a diameter $\varnothing=10 \mathrm{~cm}$ to provide an homogeneous field onto the endoluminal coil surface. As the dimension of the transmit coil is much shorter than the wavelength of the signal at $127 \mathrm{MHz}, B_{x}$ is thus directly linked to the input power $P_{i n, d B m}$ of the emission coil, via Biot and Savart equation:

$$
B_{x}=\frac{\mu_{0} I_{c}}{2} \frac{a^{2}}{\left(a^{2}+x^{2}\right)^{3 / 2}}
$$

with $a$ being the radius of the emitting loop and where $I c=$ $\sqrt{\frac{10^{\frac{P_{i n, d B m}-30}{10}}}{R_{e m}}} \cdot R_{e m}$ is the resistive load of the emission loop and takes a value of $8 \Omega$ at $127 \mathrm{MHz}$.

This emission coil is fed by a function generator providing a $\mathrm{CW}$ signal at the frequency of $127 \mathrm{MHz}$. The electrical power $P_{\text {in }}$ varies from $14 \mathrm{dBm}$ down to $-60 \mathrm{dBm}$ thanks to additional RF attenuators. The induced magnetic field at the center of the loop ranges from $700 \mathrm{nT}$ downto $140 \mathrm{pT}$.

The endoluminal coil is positioned in front of the emission coil and probe the field $B_{x}$ (see Fig. 4). It induces a rms electromotive force $\varepsilon$ onto the $z$-axis of the crystal thanks to copper deposited parallel electrodes, thus applying an electric field $E_{z}=\frac{\varepsilon}{h_{z}}, h_{z}$ being the separation distance between electrodes.

The electric field induces a modulation of the laser polarization state. This latter one can be written as a function of the input power $P_{i n, d B m}$ feeding the transmit coil and of the coupling factor $\kappa_{\text {ant }}$ between the transmit loop and the

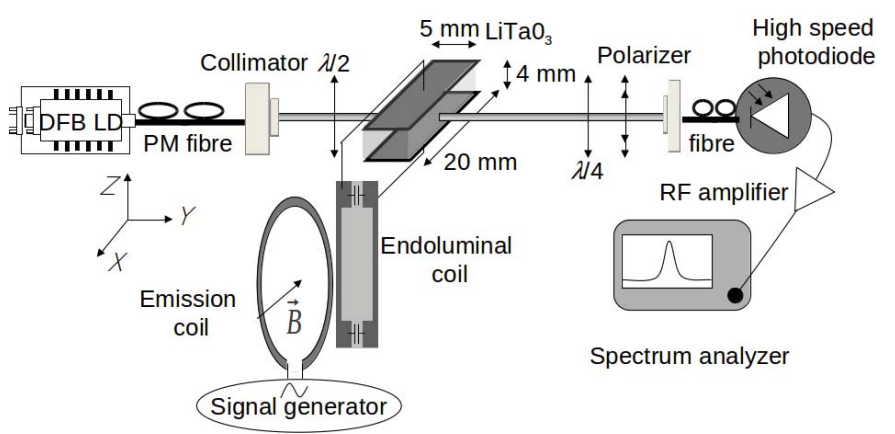

Fig. 4. Experimental bench. DFB LD: servo-controlled laser diode $(\lambda=1.55 \mu \mathrm{m})$. PM fiber: polarization maintaining fiber. $\mathrm{LiTaO}_{3}$ : Stœchiometric lithium tantalate crystal. $\lambda / 2$ and $\lambda / 4$ : half and quarter waveplates.

endoluminal coil:

$$
E_{z}\left(P_{\text {in }, d B m}\right)=\frac{\sqrt{R_{\text {load }} 10^{\frac{P_{\text {in,dBm }}+\kappa_{\text {ant }}-30}{10}}}}{h_{z}}
$$

with $R_{\text {load }}=50 \Omega$ being the resistive load of the endoluminal coil.

For $x=0$ (at the center of the loop), equations 7 and 6 lead to the link between $E_{z}$ and $B_{x}$ :

$$
E_{z}\left(B_{x}\right)=\frac{2 a 10^{\frac{\kappa_{a n t}}{20}} \sqrt{R_{\text {em }} R_{\text {load }}}}{h_{z} \mu_{0}} B_{x} .
$$

The optical source is a low noise DFB laser $(\lambda=1.55 \mu \mathrm{m}$ and Relative Intensity Noise $\left.R I N=-160 \mathrm{dBc} \cdot \mathrm{Hz}^{-1}\right)$ servo-controlled in temperature and in optical power $\left(\bar{P}_{o p t}=\right.$ $50 \mathrm{~mW}$ ). A half waveplate orients the impinging linear polarization with an angle of $45^{\circ}$ relatively to the crystal axes to maximize the conversion between the field to be measured and the polarization state modulation of the laser probe beam. The modulated polarization state is then analysed thanks to a quarter waveplate, compensating the intrinsic static birefringence of the crystal. A polarizer converts the relative dephasing $\Delta \varphi$ into a modulation of optical power $\tilde{P}_{o p t}=\frac{1}{2} \Delta \varphi \bar{P}_{\text {opt }}$. This optical modulation is finally converted into an electrical signal using an amplified $(G=37.3 \mathrm{~dB})$ pigtailed high-speed photodiode (sensitivity $\eta=0.85 \mathrm{~A} / \mathrm{W}$, loading resistance $R_{p h}=50 \Omega$ ). The mean optical power received by the photodiode after polarization treatment and fibre coupling is $\bar{P}_{o p t}=14.4 \mathrm{~mW}$. This latter signal carries the modulation and is then recorded using a spectrum analyzer.

\section{EXPERIMENTAL CHARACTERIZATION}

The analysis of the signals at different transduction stages, from the emission to the optoelectronic output is firstly investigated. Figure 5 illustrates the spectrum of the input signal feeding the transmit coil, the endoluminal coil received signal and the EO converted signal. The resolution bandwidth (RBW) of the measurement is $30 \mathrm{~Hz}$. It can be noticed on figure 5(a), that the noise floor increases by $40 \mathrm{~dB}$ after the first stage of transduction. This is due to internal attenuation of the spectrum analyser that automatically sets for high level signals. Finally, the conversion factors are $\kappa_{a n t}=-22 \pm 0.3 \mathrm{~dB}$ and 


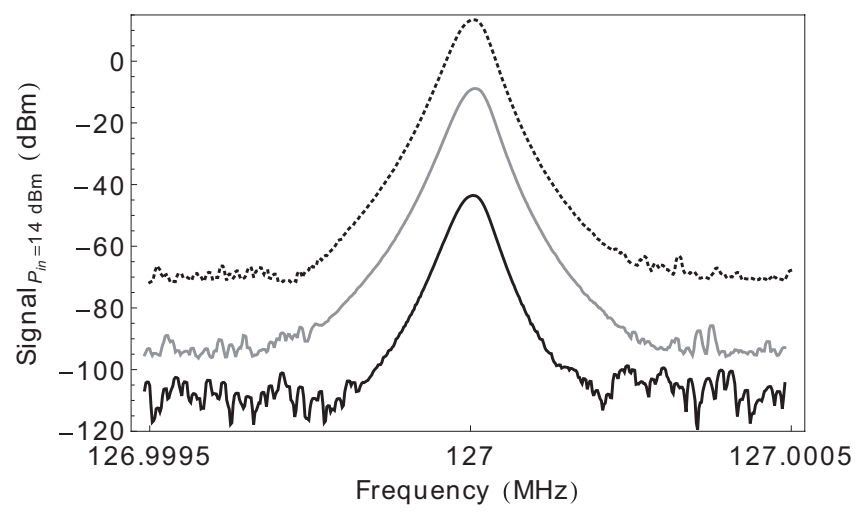

(a)

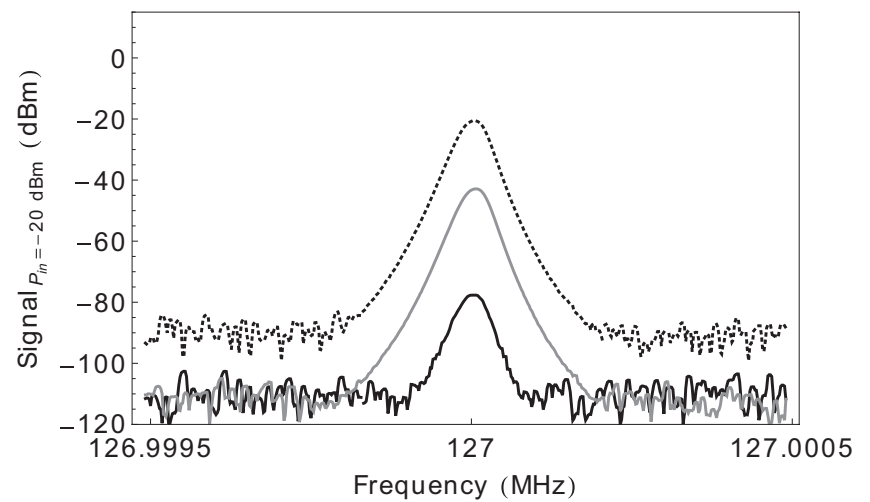

(b)

Fig. 5. Response at different stage of transduction for three values of power feeding the transmit coil. (a) $P_{\text {in }}=14 \mathrm{dBm}$. (b) $P_{\text {in }}=-20 \mathrm{dBm}$. Black dashed line: impinsging signal $P_{\text {in, dBm. }}$. Gray line: measured with the endoluminal coil. Black line: measured with the EO crystal coupled to the endoluminal coil.

$\kappa_{E O}=-34.7 \pm 0.3 \mathrm{~dB}$ for the detection coil and for the EO conversion respectively.

The sensitivity and the linearity of this conversion has been also studied over the $74 \mathrm{~dB}$ range of available power. The result is shown on figure 6 . The dynamics exceeds $60 \mathrm{~dB}$ and is down limited by the noise floor $(-112 \mathrm{dBm})$ of the spectrum analyser, which is much greater than the $R I N$ of the laser and the shot-noise on the photodiode $(-140 \mathrm{dBm}$ and $-151 \mathrm{dBm}$ in a $1 \mathrm{~Hz}$ analysis bandwidth respectively). The measurements are also compared with two curves. The linear fitting curve (gray dashed line on figure 6) is written:

$$
P_{\text {out }, \mathrm{dBm}}=A P_{\text {in }, \mathrm{dBm}}+\kappa_{\text {conv }} .
$$

$A$ is the linearity factor and is equal to 0.997. $\kappa_{\text {conv }}$ illustrates the conversion factor of the whole transduction scheme. $\kappa_{\text {conv }}=-54.1 \mathrm{~dB}$ is close to $\kappa_{\text {ant }}+\kappa_{E O}$, which is $-56.7 \pm 0.6 \mathrm{~dB}$. The second equation does not involve any fitting parameter and describes the expected output signal as a function of set-up parameters.

Introducing $\alpha_{P}=10^{\frac{G+\kappa_{\text {ant }}}{10}} R_{p h} R_{\text {load }}\left(\frac{\pi\left|\overrightarrow{\Delta K}_{x-c u t, E_{z}}\right| L_{x} \eta \bar{P}_{\text {opt }}}{\lambda_{\text {opt }} h_{z}}\right)^{2}=$ $1.2 \times 10^{-6}$, the expression between $P_{i n, d B m}$ and the output signal writes:

$$
P_{\text {out }, d B m}=10 \log _{10}\left(10^{\frac{\text { Noise }_{d B m}}{10}}+\alpha_{P} 10^{\frac{P_{\text {in }, d B m}}{10}}\right) .
$$

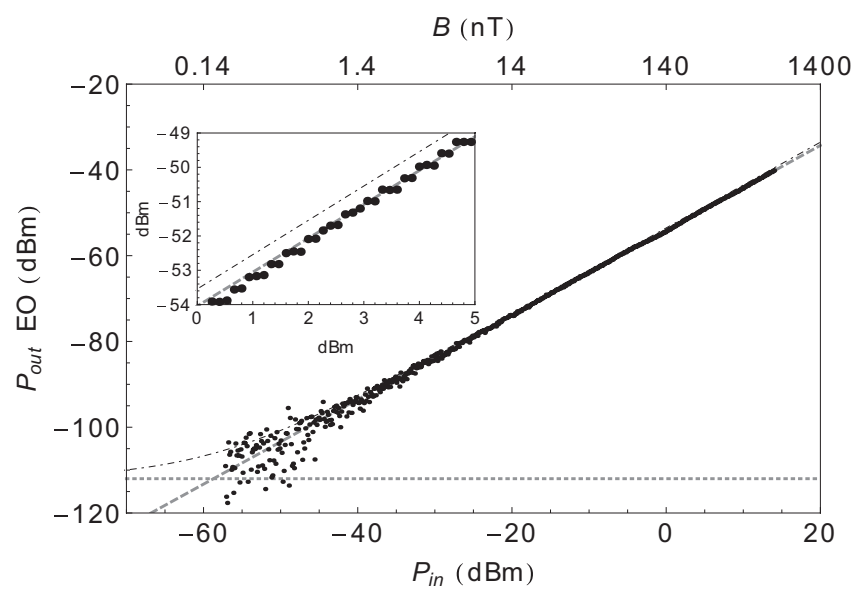

Fig. 6. EO response as a function of the input power feeding the transmit coil $(\mathrm{RBW}=100 \mathrm{~Hz})$. Top axis: input power is linked to the induced magnetic field. Black dots: measurements. Gray dashed line: linear fitting curve. Dashed dotted line: theoretical curve, including noise contributions, obtained without any fitting parameter. Horizontal gray dotted line: noise floor. Inset: zoom of the result.

The calculated value of $\alpha_{P}$ leads to an expected output signal that is fully consistent with the measured one (see inset of figure 6). Using the link between $P_{i n, d B m}$ and $B_{x}$, given in equation $6, P_{\text {out }, d B m}$ can also be written as a function of the magnetic field:

$$
P_{\text {out }, d B m}=10 \log _{10}\left(10^{\frac{\text { Noise }_{d B m}}{10}}+\alpha_{B} B_{x}^{2}\right)
$$

with $\alpha_{B}=10^{3} R_{e m}\left(\frac{2 a}{\mu_{0}}\right)^{2} \alpha_{P}=5.9 \times 10^{7} \mathrm{~mW}^{-2}$.

Moreover, the standard deviation between the measurement and the fit given by equation 9 is equal to $2.1 \mathrm{~dB}$ for the whole dynamic range and takes a very low value of $0.20 \mathrm{~dB}$ for an input signal ranging from $-26 \mathrm{dBm}$ to $14 \mathrm{dBm}$ (40 $\mathrm{dB}$ dynamic range). Finally, the minimum detectable field, which is defined by the value of the field equalizing the equation 9 and the noise floor value, is $B_{\min }=$ $176 \mathrm{pT}$. This latter value reaches $7 \mathrm{pT}$ considering only the laser RIN contribution to the noise, in a $1 \mathrm{~Hz}$ analysis bandwidth.

In order to perform an accurate and reliable measurement of the magnetic field, another relevant parameter is the stability of the experiment set-up. This stability of the output signal is mainly determined by the efficiency of the servo-control system that regulates the optical power and the laser temperature. The output signal is measured along 15 minutes for $P_{i n, d B m}=$ $14 \mathrm{dBm}$. The result is shown on figure 7(a).

The standard deviation $\sigma_{E O}$ with respect to the mean signal is deduced for the distribution represented in figure 7(b). We get $\sigma_{E O}<0.2 \mathrm{~dB}$.

\section{Magnetic Field MapPing}

In a more global use of the transduction scheme for magnetic field measurement, we here propose to evaluate the ability of the set-up to perform a near field mapping. As a characterization example of inhomogeneous magnetic field, in orientation and magnitude, we choose an asymmetric 


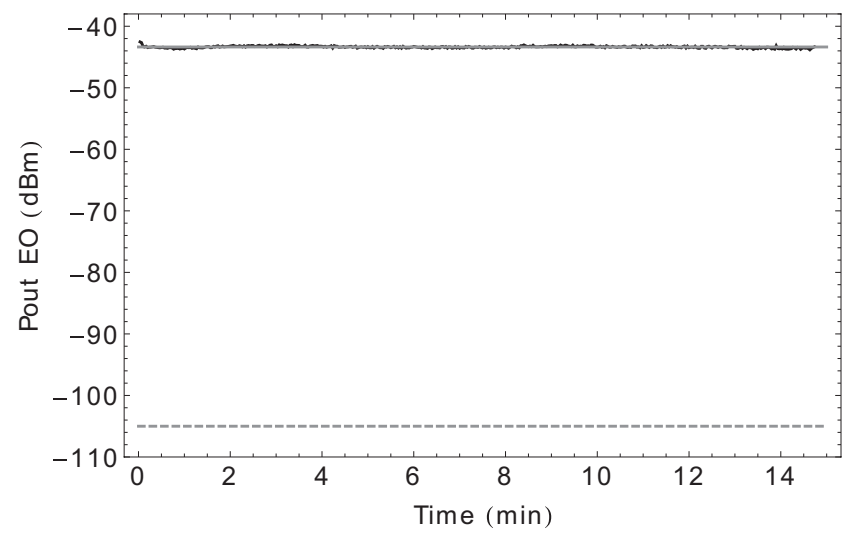

(a)

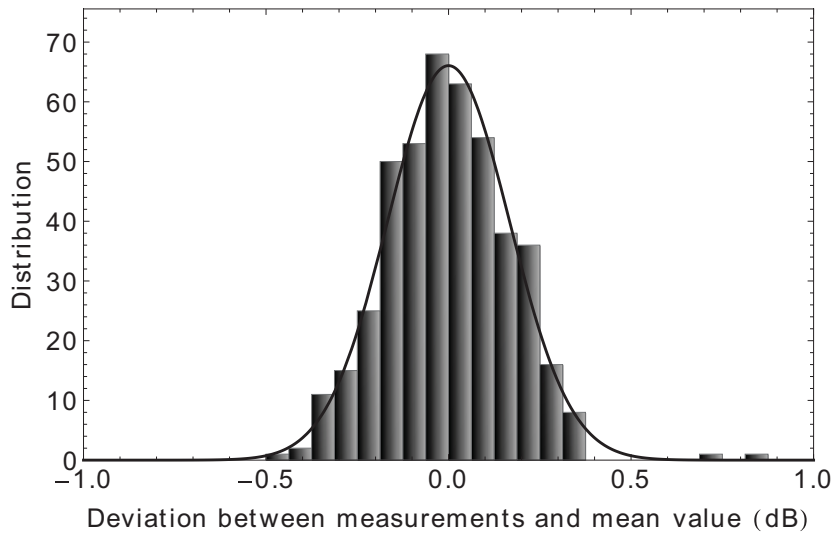

(b)

Fig. 7. (a) Black curve: stability of the EO response. Dashed gray line: average signal is indicated. Gray dotted line: noise floor $(-105 \mathrm{dBm})$. (b) Histogram: distribution of the standard deviation. Black line: associated Gaussian fit.

butterfly-shaped loop coil to be the tested device. Moreover, the typical dimensions of the butterfly-shaped loop coil are similar to the endoluminal coil and the field to be measured will be integrated on its whole radiation pattern. The device under test geometry exhibits a vanishing $B_{x}$ magnetic field at the crossing between the two loops and allows to exploit the available dynamics of the set-up. For this experiment, the referential is the same as indicated on figure 4: the $x$ axes of the two coils are aligned and the endoluminal coil is translated along the $y$ axis (see figure 8(a). The algebraic value of $B_{x}$ static magnetic field is firstly 2D mapped thanks to FDTD simulation (quickfield ${ }^{\circledR}$ ) for $z=0$. From this simulation, we define an interesting 1D path (thick arrows on figure 8(a) and 8(b)) for magnetic field mapping in order to make a comparison between measurement and simulation. Both magnetic field distributions are plotted on figure 8(c).

The experimental result exhibits a dynamics of more than $40 \mathrm{~dB}$ and is in good agreement with the simulation, especially for the spatial evolution of $B_{x}$. Nevertheless, the minimum measured value of $-82 \mathrm{~dB}$, corresponding the crossing location, is much higher than the noise floor. This is explained by the residual magnetic field surrounding the crossing point and still probed by the coil (mainly $B_{x}$ for $z \neq 0$ ). This result also demonstrates the immunity of the transducer relatively to the

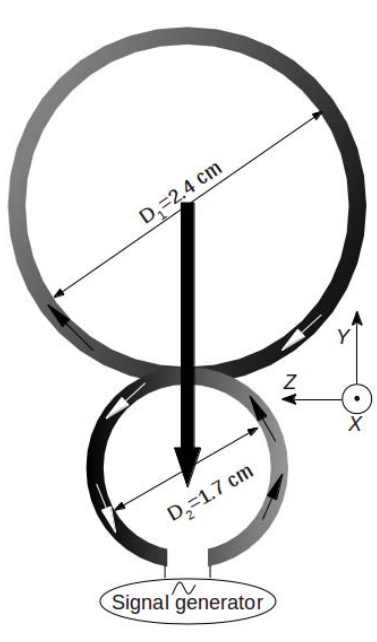

(a)

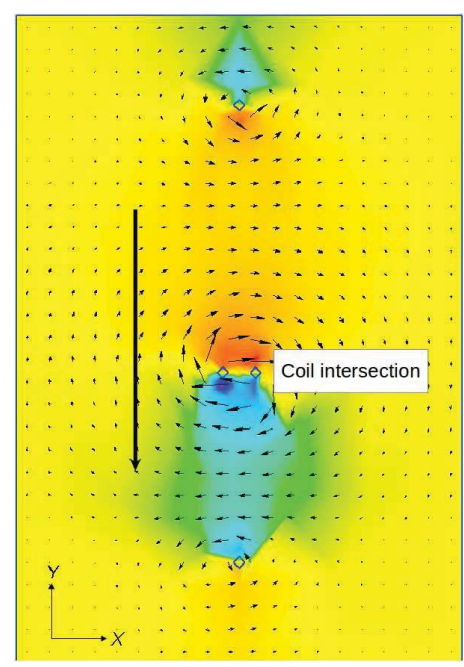

(b)

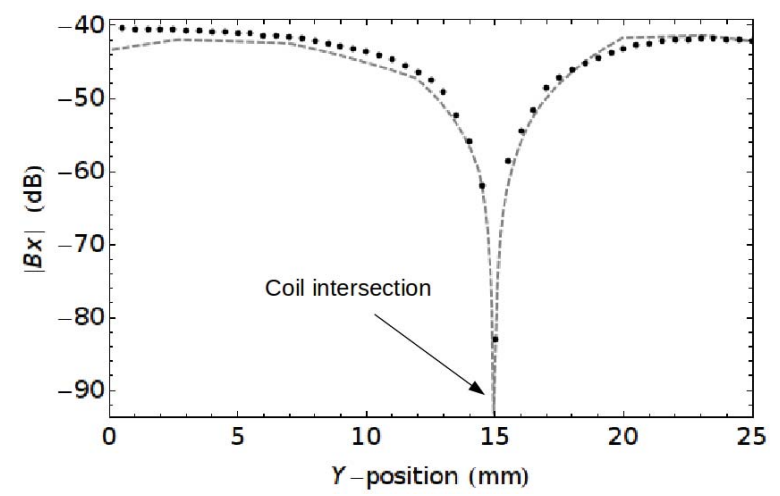

(c)

Fig. 8. (a) Schematic of the asymmetric butterfly-shaped loop coil. Thin arrows: current flow and the thick arrow is the mapping path. (b) FDTD simulation of the butterfly-shaped loop coil. Color scale from blue: lowest negative $B_{x}$. To red: highest positive $B_{x}$. Down arrow: measurement location. (c) Spatial distribution of the magnetic field component $B_{x}$ along the $y$-axis of the asymmetric loop coil. Black dots: measured with EO transducer. Dashed gray line: simulated with FDTD.

transverse component $\left(B_{y}\right)$ of the magnetic field and illustrate the vectorial behaviour of the sensor.

\section{CONCLUSION}

A $\mathrm{LiTaO}_{3}$ crystal is used together with an endoluminal coil to demonstrate the ability of such transducer to perform a remote and reliable characterization of RF magnetic field at 3T NMR frequency. The whole transducing scheme has been theoretically studied. The in-lab implementation demonstrates a very good agreement with the expected results. The experimental minimum detectable field is weaker than $1 \mathrm{nT}$. The accuracy of the measured signal is better than $0.5 \mathrm{~dB}$ along a $50 \mathrm{~dB}$ dynamic range with a rather good stability (deviation lower than $0.2 \mathrm{~dB}$ for a 15 minutes temporal window). The previous mentioned performances allow to map one component of the magnetic field and a vectorial selectivity greater than $40 \mathrm{~dB}$ is demonstrated. Development of such sensor scheme constitutes a first step towards a fully pigtailed probe. This technique would also benefit from the potential miniaturization of the crystal size $\left(h_{z} \simeq 10 \mu \mathrm{m}\right.$ for an $\mathrm{EO}$ 
waveguide), in order to increase the sensitivity (by a factor 400). Furthermore, optical amplifier ( $+24 \mathrm{~dB}$ typically) can be used to increase the optical modulation after polarization treatment to reach lower minimum detectable field, required for actual MRI system, while keeping advantages of passive and remote EO configuration.

\section{REFERENCES}

[1] M. Armenean, O. Beuf, F. Pilleul, and H. Saint-Jalmes, "Optimization of endoluminal loop radiofrequency coils for gastrointestinal wall MR imaging," IEEE Sensors J., vol. 4, no. 1, pp. 57-64, Feb. 2004.

[2] O. Beuf, F. Pilleul, M. Armenean, G. Hadour, and H. Saint-Jalmes, "In vivo colon wall imaging using endoluminal coils: Feasibility study on rabbits," J. Magn. Reson. Imag., vol. 20, no. 1, pp. 90-96, 2004.

[3] C. Armenean, E. Perrin, M. Armenean, O. Beuf, F. Pilleul, and H. SaintJalmes, "RF-induced temperature elevation along metallic wires in clinical magnetic resonance imaging: Influence of diameter and length," Magn. Reson. Med., vol. 52, no. 5, pp. 1200-1206, 2004.

[4] J. Yuan, J. Wei, and G. X. Shen, "A 4-channel coil array interconnection by analog direct modulation optical link for 1.5-T MRI," IEEE Trans. Med. Imag., vol. 27, no. 10, pp. 1432-1438, Oct. 2008.

[5] O. G. Memis, Y. Eryaman, O. Aytur, and E. Atalar, "Miniaturized fiberoptic transmission system for MRI signals," Magn. Reson. Med., vol. 59, no. 1, pp. 165-173, 2008.

[6] S. Fandrey, S. Weiss, and J. Müller, "Miniature optical signal transmission system for an active intravascular device," Proc. Int. Soc. Magn. Reson. Med., vol. 15, p. 444, May 2007.

[7] C. Du, J. Yuan, and G. X. Shen, "Comparison of FP, VCEL and DFB laser diode in optical transmission for MR RF coil array," Proc. Int. Soc. Magn. Reson. Med., vol. 15, p. 1041, May 2007.

[8] E. Suzuki, S. Arakawa, H. Ota, K. I. Arai, and R. Sato, "Optical magnetic field probe with a loop antenna element doubly loaded with electrooptic crystals," IEEE Trans. Electromagn. Compat., vol. 46, no. 4, pp. 641-647, Nov. 2004.

[9] W.-K. Kuo and J.-Y. Kuo, "High sensitivity electro-optic magnetic field probe using Fabry-Pérot resonance," Microw. Opt. Technol. Lett., vol. 50, no. 12, pp. 3212-3214, 2008.

[10] J. N. Blake, "Fiber optic interferometric circuit and magnetic field sensor," U.S. Patent 5644397, Jul. 1, 1997.

[11] A. Mhapatra and V. E. Stenger, "Electro-optic electromagnetic field sensor system with optical bias adjustment," U.S. Patent 5963034, Oct. 5, 1999.

[12] L. Duvillaret, S. Rialland, and J.-L. Coutaz, "Electro-optic sensors for electric field measurements. II. Choice of the crystals and complete optimization of their orientation," J. Opt. Soc. Amer. B, vol. 19, no. 11, pp. 2704-2715, 2002.

[13] L. Sun, S. Jiang, and J. R. Marciante, "All-fiber optical magnetic-field sensor based on Faraday rotation in highly terbium-doped fiber," Opt. Exp., vol. 18, no. 6, pp. 5407-5412, 2010.

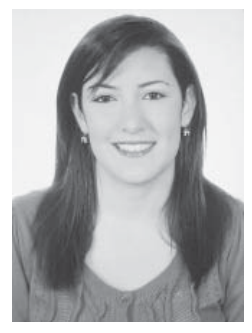

Reina Aydé was born in Lebanon in 1988. She received the M.Sc. degree in physics of Nano Science and Functional Materials from the Lebanese University Faculty of Science II, Beirut, Lebanon, in 2010. She is currently pursuing the Ph.D. degree in electro-optic microsystem for MR endoluminal coil' with the CREATIS Laboratory, University of Lyon 1, Lyon, France.

She is working in collaboration with IMEPLAHC Laboratory, University of Savoie and Kapteos Company.

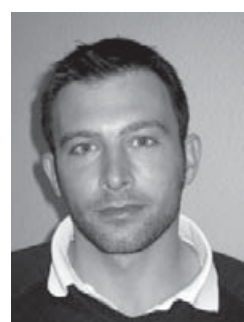

Gwenaël Gaborit was born in Lens, France, in 1978. He received the M.Sc. degree in microwaves and microelectronics from the University of Lille, Lille, France, in 2002, and the Ph.D. degree in physics from the University of Savoie, Savoie, France, in 2005.

$\mathrm{He}$ has been an Associate Professor with the IMEP-LAHC Laboratory, since 2006. He is cofounder of the Kapteos Company and is currently the Chief Science Officer. He is involved in $\mathrm{THz}$ spectroscopy and plasmonic. His current research interests include developing electro-optic sensors dedicated to ultra wide band and noninvasive electric field measurement.

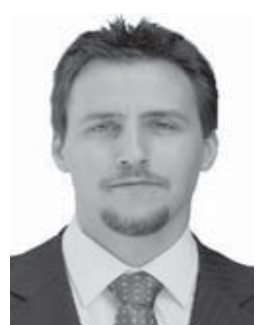

Pierre Jarrige was born in Brive-La-Gaillarde, France, in 1981. He received the Master's degree in laser, matter and biological media from the University of Paris-VI, Paris, France, in 2007. He is currently pursuing the Ph.D. degree with the Grenoble Institute of Technology, Grenoble, France.

$\mathrm{He}$ is currently with the IMEP-LAHC Laboratory. His current research interests include development of electro-optic sensors devoted to bioelectromagnetism studies, including SAR measurements.

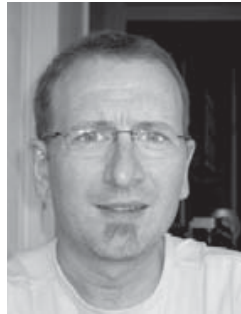

Lionel Duvillaret was born in Thonon-les-bains, France, in 1966. He received the Ph.D. degree in physics from the University of Paris XI, Orsay, France, in 1994.

He was involved in research on electrooptic sampling with the Institute of Fundamental Electronics, Orsay, from 1990 to 1994. From 1993 to 2005, he was an Assistant Professor of physics working on spectroscopy, electrooptic characterization of electric fields, and electromagnetic bandgap materials with the LAHC Laboratory, University of Savoie, Le Bourget-du-Lac, France. Since 2005, he has been a Professor of physics with IMEP-LAHC Laboratory, Grenoble Institute of Technology, Grenoble, France. In November 2009, he has founded Kapteos SAS, Le Bourget-du-Lac, and is currently the Chief Executive Officer. He is the author or co-author of over 125 journal papers and international conference proceedings and is the author of seven patents.

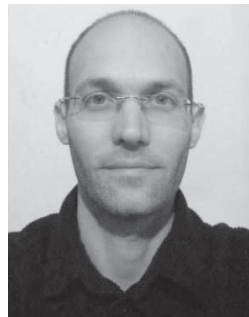

Raphaël Sablong was born in France in 1975 He received the Ph.D. degree in physics from the University of Grenoble, Grenoble, France, in 2001.

He was a Post-Doctoral fellow with the Laboratoire de Physique des Lasers, University of Paris XIII, Paris, France, from 2001 to 2002, working on modeling and device of near-infrared reflectance spectroscopy for muscle oximetry. He is currently an Assistant Professor with the University of Lyon, Lyon, France, and a member of the NMR and Optics: Methods and Systems research team from the CNRS 5220, Laboratoire Creatis. His current research interests include photon migration in scattering media, optical instrumentation development for biomedical diagnosis. 


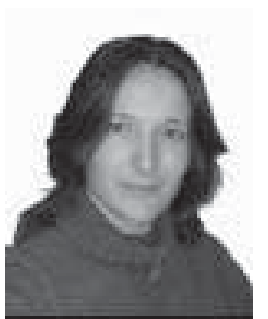

Anne-Laure Perrier was born in France in 1980. She received the M.Sc. degree in optics, optoelectronics, and microwaves from the Institut National Polytechnique de Grenoble, Grenoble, France, in 2003, and the Ph.D. degree from the Laboratory of Microwaves and Characterization, University of Savoie, Savoie, France, in 2006.

She has been an Assistant Professor with Claude Bernard University, Lyon, France, since September 2008, where she teaches electronics and signal processing. She continues the research with the Research Center on Medical Imaging. She designs and realizes RF sensors for magnetic resonance imaging applications. Her current research interests include theory, design, and realization of tunable-impedance transformers and tunable power dividers.

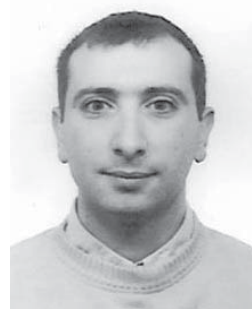

Olivier Beuf was born in France in 1970. He received the Ph.D. degree in physics from the University de Lyon, Lyon, France, in 1998.

He was a Post-Doctoral fellow with the University of California, San Francisco, from 1998 to 2000 , working on advanced methodological MRI developments for musculoskeletal applications. He was a MRI Application Specialist with Siemens Medical Solutions, France. He joined the CNRS 5012 NMR Laboratory in 2000. He is the head of the NMR and Optics: Methods and Systems research team from the CREATIS Laboratory. His current research interests include radiofrequency coils and sequence developments for medical or biological MRI applications both on human and small animals. 